UCF-CM-95-002

\title{
Integer quantum Hall effect for hard-core bosons and a failure of bosonic Chern-Simons mean-field theories for electrons at half-filled Landau level
}

\author{
O. Heinonen and M.D. Johnson \\ Department of Physics, University of Central Florida, Orlando, FL 32816-2385
}

\begin{abstract}
Field-theoretical methods have been shown to be useful in constructing simple effective theories for two-dimensional (2D) systems. These effective theories are usually studied by perturbing around a mean-field approximation, so the question whether such an approximation is meaningful arises immediately. We here study 2D interacting electrons in a half-filled Landau level mapped onto interacting hard-core bosons in a magnetic field. We argue that an interacting hard-core boson system in a uniform external field such that there is one flux quantum per particle (unit filling) exhibits an integer quantum Hall effect. As a consequence, the mean-field approximation for mapping electrons at halffilling to a boson system at integer filling fails. (Contact mdj@physics.ucf.edu. Cond-mat paper cond-mat/9510122.)

73.40.Hm
\end{abstract}

Typeset using REVTEX 
Chern-Simons (CS) field theoretical approaches to the fractional (and integer) quantum Hall effect (FQHE and IQHE, respectively) developed recently [1] provide simple effective theories for electron FQHE and IQHE systems. In these approaches, the physical electron system is mapped by a singular gauge transformation onto an equivalent system of fermions or bosons interacting with a statistical CS gauge field. The equivalent system is then typically studied by first introducing a mean-field approximation, in which the particles experience a constant net field which is the sum of the external field and the average CS field, and then by perturbing around this mean-field approximation. Since the CS field is singular, it may at first seem surprising that the rather crude mean-field approximation makes sense at all, and it is essential to understand when this approximation works, i.e. when perturbation about the mean-field converges to the physical system, or at least gives physical results, and when it breaks down. The key to success of the mean-field approximation as a suitable starting point seems to be when both the physical electron system and the equivalent CS system have energy gaps. At the very least, perturbation about the meanfield then makes sense, since the energy gap suppresses fluctuations about the mean-field, although there is no guarantee that perturbation theory converges to the physical system. When both the physical system and the equivalent CS system are gapless, sensible results can be obtained from the mean-field approximation, although infrared divergences may show up [2] and some renormalization scheme has to be devised. The purpose of this paper is add to our understanding of the applicability of CS theories by giving an example for which we argue that the mean-field approximation is not a suitable starting point at all: a half-filled Landau level mapped onto CS interacting hard-core bosons at one filled Landau level. For this case, we will argue that perturbation theory about the mean-field does not converge to the physical system at all. This is because the mean-field approximation describes a system of interacting hard-core bosons at integer filling. Jain and Rao [3] have recently suggested that non-interacting bosons at $\nu=1$ have an energy gap and exhibit an integer quantum Hall effect, and we argue that this holds for interacting hard-core bosons. This gap remains to all orders in perturbation theory, while the real electron system is gapless. 
Jain [4] has recently shown that mapping the electron system onto CS fermions provides a natural way to study the FQHE. In this mapping, one starts with a system of twodimensional (2D) electrons of density $n$ in an external field $A_{\mu}$ such that the filling factor $\nu=2 \pi n \ell_{B}^{2}=p /(2 n p+1)$, with $B_{\mu}=\epsilon_{\mu \nu \lambda} \partial_{\nu} A_{\lambda}$ and the magnetic length $\ell_{B}^{2}=c /(e B)$, and $p$ and $n$ integers. (We will use units in which $\hbar=c=1$ and notation in which Greek indices denote time and space dimension, and Roman indices denote space dimension with an implicit flat Minkowski metric $g_{\mu \nu}$. Summation over Greek indices will be implied unless stated otherwise.) One then performs a singular gauge transformation by attaching flux tubes with an even number $2 n$ of flux quanta to each electron, which transforms the system into an equivalent one of fermions in an external field $A_{\mu}$ plus the Chern-Simon field $a_{\mu}$ from the flux tubes. The resulting equivalent system can then be studied by starting with a mean-field approximation (the saddle-point approximation in a Lagrangian formulation), in which external and statistical magnetic fields are taken to be uniform at their spatial average. The mean-field system thus consists of fermions in an average magnetic field at integer filling $\nu=p$. Fluctuations in the statistical field can then be added for example within a random-phase approximation in a Hamiltonian formulation, or the equivalent oneloop approximation in a Lagrangian formulation by expanding the fields up to second order about the saddle-point and then integrating out the fermion fields. It is expected that perturbation theory, such as the one-loop expansion, about the saddle point in this case gives a good description of the low-lying excitations of the real system (except for the magnetoroton minimum) [5]. The reason is that the saddle-point approximation describes an IQHE system, which has an energy gap. This gap is the cyclotron energy of the net average magnetic field which has contributions from the average statistical field and the external field. One can then formally integrate out the high-energy modes to obtain an effective theory of a massive fermion field coupled to a CS field. It is well-known that the statistical parameter (the topological mass term) of a Chern-Simons field coupled to a massive scalar or spinor field does not renormalize beyond the one-loop level, at which level it receives at the most a small correction [6]. Therefore, the energy gap remains nonzero 
and finite to all orders in perturbation theory. Furthermore, topological theorems $\llbracket 7$ for the Hall conductivity ensure that the statistical parameter does not renormalize at all for IQHE and FQHE systems, so that the incompressible system indeed has a quantized Hall conductivity. Note that it is crucial that the average magnetic field at the the saddle-point approximation is not entirely due to the statistical field itself - if this were the case, the one-loop approximation would give rise to a compressible system due to exact cancellation between the Hall conductivity of the CS fermions in the constant average field and the statistical parameter of the CS term in the Lagrangian. This is precisely the case for anyon superconductors [8], where one starts with anyons in no external field and transform to CS fermions or hard-core bosons in a net field due entirely to the CS field.

On the other hand, an FQHE system can be mapped onto a system of hard-core CS bosons [5,9] by performing a singular gauge transformation which attaches flux tubes with an odd number $(2 p+1)$ of flux quanta to the electrons. At the mean-field level, one then has a boson system in a uniform magnetic field. In general, such a system is compressible and contains vortices, which are the locations where the boson order parameter vanishes, and these generate an additional gauge field which has to be included [10,[1]. However, at special fillings $\nu=1 /(2 p+1)$, i.e., such that the average magnetic field precisely vanishes (the average Chern-Simon gauge field precisely cancels the external gauge field), there are no vortices and the boson system is in fact incompressible (see below). In this case, the average vortex density vanishes [10] and the vortices acquire a finite mass. One can proceed to formally integrate out the bose fields, and assume [5] that the statistical parameter of the CS term does not renormalize. The result is an effective Lagrangian for the fluctuations in the CS field with only massive modes and a quantized Hall conductance. This non-trivial result has also been obtained rigorously by Read [9] starting with the Laughlin FQHE wavefunctions. The reason that the statistical parameter does not renormalize (even though the mean-field approximation describes a system of hard-core bosons in zero magnetic field, which one would think is gapless) is that the FQHE states have broken $U(1)$ symmetry described by off-diagonal long-range order in a charged Bose field $\phi$ (the Bose field condenses 
into a superfluid). The massless Goldstone mode which appears in the broken-symmetry phase due to phase fluctuations in the order parameter vanishes by the standard AndersonHiggs mechanism when the system is coupled to the CS statistical field. Therefore, the effective CS Lagrangian has only massive modes.

Recently, electron systems at half filling, $\nu=1 / 2$, have been studied experimentally [12 14 and theoretically. Experimentally, these systems are gapless metals and show a finite longitudinal resistivity $\rho_{x x}$ with a sharp minimum as a function of filling factor precisely at $\nu=1 / 2$, while the Hall resistivity $\rho_{x y}$ is not quantized but rather shows its classical form $\rho_{x y} \sim B /($ nec $)$. Careful experiments indicate [12, 14] that in the presence of disorder, $\nu=1 / 2$ is the critical point for a transition between an incompressible quantum Hall fluid and an weakly localized Anderson insulator. Theoretically, $\nu=1 / 2$ has been studied by mapping the electrons onto CS fermions in zero average field [15,2, 16], as well as by other more traditional approaches [20]. Kalmeyr and Zhang [15] showed that within the CS theory, disorder in the effective system of 2D CS fermions in zero average magnetic field leads to density fluctuations which couple to fluctuations in the statistical field. This results in a fluctuating net magnetic field which breaks the time-reversal invariance responsible for weak localization in two dimensions, and the system shows a metallic conductivity. In a very comprehensive and creative work, Halperin, Lee and Read [2] investigated interacting CS fermions in zero average magnetic field, with or without weak disorder. Under the assumption that the system exhibits a Fermi surface at the mean-field level, they went on to study the effective mass renormalization, in addition to various experimental signatures. Even though infrared divergences at the one-loop level for short-range interactions lead to infinite renormalization of the effective mass, while Coulomb interactions lead to logarithmic corrections, they argued that the theory is renormalizable and that the interacting system is Fermi-liquid like (or is a marginal Fermi liquid). This has recently been demonstrated in first-principle numerical calculations by Rezayi and Read [16], and there is also recent experimental evidence of a Fermi surface [17-19]. It is clear that for this system, perturbation theory about the mean-field system has to be done with extreme care - at the mean-field 
level, the system is gapless (even though it is incompressible for Coulomb interactions! [2]) and there is nothing which can guarantee the stability of the one-loop approximation and zero renormalization of the statistical parameter beyond the one-loop level. Therefore, one can expect heavy, perhaps infinite, renormalization of all physical parameters, including the statistical parameter.

We will here study interacting electrons at $\nu=1 / 2$ in zero or weak disorder by mapping the system onto a system of CS hard-core bosons at filling $\nu=1$ by attaching a flux tube of one flux quantum to each electron. At the saddle-point, this is then a system of hard-core bosons at filling $\nu=1$. Jain and Rao [3] have recently argued that non-interacting hard-core bosons exhibit a $\nu=1$ IQHE in the thermodynamic limit. We will here argue that this holds when interactions are included. As a consequence, perturbation theory to infinite order about the saddle point is renormalizable and converges, but not to the physical system. We argue that this is due to the non-analyticity of the true ground-state wavefunction, while the mean-field wavefunction is analytical and outside the radius of convergence of perturbation theory about the true ground state.

We start with a system of $2 \mathrm{D}$ electrons at half filling $\nu=1 / 2$ in the external field $A_{\mu}$. The system is described by the action

$$
\begin{aligned}
S= & \int d^{2} r d t\left\{\psi^{\dagger}(\mathbf{r}, t)\left[i \partial_{0}-e A_{0}-\mu\right] \psi(\mathbf{r}, t)-\frac{1}{2 m} \psi^{\dagger}(\mathbf{r}, t)[-i \nabla-e \mathbf{A}]^{2} \psi(\mathbf{r}, t)\right\} \\
& -\frac{1}{2} \int d^{2} r d t \int d^{2} r^{\prime} r d t^{\prime}\left(\psi^{\dagger}(\mathbf{r}, t) \psi(\mathbf{r}, t)-n_{0}\right) V\left(\left|\mathbf{r}-\mathbf{r}^{\prime}\right|\right)\left(\psi^{\dagger}\left(\mathbf{r}^{\prime}, t^{\prime}\right) \psi\left(\mathbf{r}^{\prime}, t^{\prime}\right)-n_{0}\right),
\end{aligned}
$$

where $n_{0}$ is the average density; $2 \pi n_{0} \ell_{B}^{2}=\nu=1 / 2$. By performing a singular gauge transformation which attaches a flux quantum to each electron we obtain the Bose action

$$
\begin{aligned}
S_{B}= & \int d^{2} r d t\left\{\phi^{\dagger}(\mathbf{r}, t)\left[\partial_{0}-e A_{0}-e a_{0}-\mu\right] \phi(\mathbf{r}, t)-\frac{1}{2 m} \phi^{\dagger}(\mathbf{r}, t)[-i \nabla-e \mathbf{A}-e \mathbf{a}]^{2} \phi(\mathbf{r}, t)\right\} \\
& +\frac{1}{2} \int d^{2} r d t \int d^{2} r^{\prime} r d t^{\prime}\left(\phi^{\dagger}(\mathbf{r}, t) \phi(\mathbf{r}, t)-n_{0}\right) V\left(\left|\mathbf{r}-\mathbf{r}^{\prime}\right|\right)\left(\phi^{\dagger}\left(\mathbf{r}^{\prime}, t^{\prime}\right) \phi\left(\mathbf{r}^{\prime}, t^{\prime}\right)-n_{0}\right) \\
& +\frac{\theta e^{2}}{4} \epsilon_{\mu \nu \lambda} a_{\mu} f_{\nu \lambda .} .
\end{aligned}
$$

Here, the CS field $a_{\mu}$ is given by 


$$
\mathbf{a}(\mathbf{r})=-\frac{c}{2 \pi \theta e} \int d^{2} r^{\prime} \frac{\hat{\mathbf{z}} \times\left(\mathbf{r}-\mathbf{r}^{\prime}\right)}{\left|\mathbf{r}-\mathbf{r}^{\prime}\right|^{2}} \phi^{*}\left(\mathbf{r}^{\prime}\right) \phi\left(\mathbf{r}^{\prime}\right)
$$

with $\theta=1 /(2 \pi)$. We change to the Euclidean imaginary-time action

$$
\begin{aligned}
S_{\tau}= & \int d^{2} r d \tau\left\{\phi^{\dagger}(\mathbf{r}, \tau)\left[\partial_{\tau}-e A_{0}-e a_{0}-\mu\right] \phi(\mathbf{r}, \tau)+\frac{1}{2 m} \phi^{\dagger}(\mathbf{r}, \tau)[-i \nabla-e \mathbf{A}-e \mathbf{a}]^{2} \phi(\mathbf{r}, \tau)\right\} \\
& +\frac{1}{2} \int d^{2} r d \tau \int d^{2} r^{\prime} r\left(\phi^{\dagger}(\mathbf{r}, \tau) \phi(\mathbf{r}, \tau)-n_{0}\right) V\left(\left|\mathbf{r}-\mathbf{r}^{\prime}\right|\right)\left(\phi^{\dagger}\left(\mathbf{r}^{\prime}, \tau\right) \phi\left(\mathbf{r}^{\prime}, \tau\right)-n_{0}\right) \\
& +\frac{\theta e^{2}}{4} \epsilon_{\mu \nu \lambda} a_{\mu} f_{\nu \lambda} .
\end{aligned}
$$

and consider the partition function

$$
\mathcal{Z}=\int \mathcal{D} \phi^{*} \mathcal{D} \phi \mathcal{D} a_{\mu} \exp \left[-S_{\tau}\right]
$$

where the path-integrals are to be understood as coherent-states path integrals. We proceed by formally integrating out the bose fields with the CS fields $a_{\mu}$ fixed near their saddle-point values $a_{\mu}^{0}, \nabla \times \mathbf{a}^{0}=-2 \pi n_{0} / e$, to obtain the partition function

$$
\mathcal{Z}_{0}\left(a_{\mu}\right) \int \mathcal{D} a_{\mu} \exp \left[-S_{\mathrm{CS}}\left(a_{\mu}\right)\right]
$$

Here, $S_{\mathrm{CS}}\left(a_{\mu}\right)$ is the Euclidean Chern-Simons action. Next, we expand the fields $a_{\mu}$ up to quadratic order about their saddle-point values $a_{\mu}^{0}$. The result is an effective partition function $\mathcal{Z}_{\text {eff }}\left(a_{\mu}^{0}\right)$ :

$$
\mathcal{Z}_{\text {eff }}\left(a_{\mu}^{0}\right)=\mathcal{Z}_{0}\left(a_{\mu}^{0}\right) \int \mathcal{D} a_{\mu}^{\prime} \exp \left[-S_{\text {eff }}\left(a_{\mu}^{\prime}\right)\right]
$$

The effective action $S_{\text {eff }}\left(a_{\mu}^{\prime}\right)$ describes the quadratic fluctuations of the CS field in a medium of hard-core bosons at a fixed magnetic field $\nu=1$ and is given formally by

$$
S_{\mathrm{eff}}\left(a_{\mu}^{\prime}\right)=\int d^{2} r d \tau \frac{1}{2}\left[a_{\mu}^{\prime}\right]^{*} \Pi_{\mu \nu} a_{\nu}^{\prime}+\frac{\theta}{4} \epsilon_{\mu \nu \lambda} a_{\mu}^{\prime} f^{\prime}{ }_{\nu \lambda}
$$

where $\Pi_{\mu \nu}$ is the current-current correlation function of the interacting hard-core boson system at $\nu=1$,

$$
\Pi_{\mu \nu}\left(\mathbf{r}, t ; \mathbf{r}^{\prime}, t^{\prime}\right)=\left\langle j_{\mu}(\mathbf{r}, t) j_{\nu}\left(\mathbf{r}^{\prime}, t^{\prime}\right)\right\rangle
$$


In general, $\Pi_{\mu \nu}$ is very complicated and describes properties such as the dielectric function, magnetic susceptibility, and Hall conductivity $\sigma_{x y}^{0}$, and we don't know much at all about $\Pi_{\mu \nu}$. Except, as we now argue, that this Bose system has an energy gap and that its Hall conductivity is quantized at $\sigma_{x y}^{0}=e^{2} /(4 \pi)$. Jain and Rao [3] have recently suggested that non-interacting hard-core bosons may exhibit IQHE at $\nu=1$ in the thermodynamic limit. This may seem surprising, for the following reason. Decompose the interaction potential $V\left(r-r^{\prime}\right)$ in relative angular momentum (RAM) components, the strength of which are given by the pseudo-potential parameters $V_{0}, V_{2}, \ldots$, where $V_{2 n}$ denotes the interaction energy of two bosons with RAM $2 n$. Note that the total wavefunction is even under interchange of two bosons, so that no two bosons can have odd RAM. The parameter $V_{0}$ describes the hard-core interaction, since the zero RAM channel is the only one which allows the bosons to be at the same place. We assume that $V_{0} \gg \hbar \omega_{c} \gg V_{2}>V_{4}>\ldots$ In the $V_{0}$ approximation, we can construct a Jastrow wavefunction in which all bosons avoid zero RAM. With all bosons in the lowest Landau level, we must have $\nu \leq 1 / 2$ in order to avoid RAM 0. By occupying the next Landau level, we can reach fillings $\nu \leq 2 / 3$ and avoiding RAM 0, and so on. Simple trial wavefunctions corresponding to these fillings can be constructed by starting with the wavefunctions for non-interacting electrons at $\nu=p$ and by multiplying the wavefunction by the Jastrow factor $\Pi\left(z_{i}-z_{j}\right)$. This generates hard-core boson FQHE at fillings $\nu=p /(p+1)$, with $\nu=1$ as an accumulation point, similar to $\nu=1 / 2$ for electrons. Consequently, one does not expect a boson QHE at $\nu=1$. However, as $\nu \rightarrow 1$ in this construction, there is a finite fraction of particles in all Landau levels, and the energy cost becomes very large. Jain and Rao suggested instead another variational scheme for boson wavefunctions, in which the wavefunction is obtained from the product of two fermion wavefunctions $\Psi_{p}$ and $\Psi_{p^{\prime}}$, each of which describes $p$ and $p^{\prime}$ filled electron Landau levels, respectively. For the case of non-interacting hard-core bosons at $\nu=1$ the boson wavefunction $\Phi_{1}$ is then constructed from two fermion wavefunctions for filled two lowest Landau levels. The fermion wavefunction for two filled Landau levels can be written $\Psi_{2}=f_{2}([z, z *]) \exp \left[-\sum_{i}\left|z_{i}\right|^{2} / 4\right]$. Here, $f_{2}([z, z *])$ is an antisymmetric polynomial in the co- 
ordinates $z_{i}=x_{i}+i y_{i}$, and $[z, z *]$ denotes the collections $\left\{z_{i}\right\}$ and $\left\{z_{i}^{*}\right\}$. The resulting boson wavefunction $\Phi_{1}=f_{2}^{2} \exp \left[-\sum_{i}\left|z_{i}\right|^{2} / 4\right]$ at the very least gives a rigorous upper bound to the ground state energy of bosons at $\nu=1$ which is much lower than the previous one. Although we have no formal proof, we will assume that $\Phi_{1}$ is in fact the lowest-energy wavefunction at $\nu=1$ which avoids RAM 0. This assumption can be supported by the following argument. From the single-particle Landau level lowering operator $\hat{a} \equiv 1 / \sqrt{2}\left(z / 2+2 \partial / \partial z^{*}\right)$ and angular momentum lowering operator $\hat{b} \equiv 1 / \sqrt{2}\left(z^{*} / 2+2 \partial / \partial z\right)$ (we are using the symmetric gauge) we construct many-body Landau level lowering and angular momentum lowering operators $\hat{A}=\sum_{i} \hat{a}_{i}$ and $\hat{B}=\sum_{i} \hat{b}_{i}$ acting on the many-body wavefunctions. A straightforward calculation then yields $\hat{A} \Phi_{1}=\hat{B} \Phi_{1}=0$. This is because both operators act as derivative operators on $f_{2}^{2}$, which by construction is the the square of the polynomial part of an electronic wavefunction of two filled Landau levels. The net effect is then the same as having two Landau levels filled with electrons and attempting to two lower the total angular momentum or to send all electrons to a lower Landau level, both of which give zero. We conclude that $\Phi_{1}$ cannot be obtained by operating with any analytic function of $\hat{A}^{\dagger}$ and $\hat{B}^{\dagger}$ on some other wavefunction, and so is a good candidate for the ground state.

Within this variational scheme the state $\Phi_{1}$ has an energy gap of order $\hbar \omega_{c}$ and so describes an IQHE. This ground state and its energy gap are robust under adiabatic turningon of a finite number of RAM components $V_{2 n}$ since $\hbar \omega_{c} \gg V_{2 n}$. Using arguments due to Laughlin [21] and Halperin [22], it is straightforward to demonstrate that this IQHE survives weak disorder, and that $\sigma_{x y}^{0}$ is quantized at $\sigma_{x y}^{0}=e^{2} /(2 \pi)$. As a consequence of this, the effective statistical parameter $\theta_{\text {eff }}$ becomes [23]

$$
\frac{1}{\theta_{\mathrm{eff}}}=\frac{1}{\sigma_{x y}^{0}}+\frac{1}{\theta}
$$

or

$$
\theta_{\mathrm{eff}}=\frac{e^{2}}{4 \pi}
$$

The energy gap at the saddle-point approximation ensures that the amplitude of field fluctuations about their saddle-point values are small, so a gradient expansion of $\Pi_{\mu \nu}$ makes 
sense to obtain the effective action for low-lying excitations. Since $\theta_{\text {eff }} \neq 0$, the low-lying modes are massive. In other words, if we were to start by intergrating out the short-distance fluctuations in the Bose field, we would obtain an effective theory describing a massive charged scalar field interacting with the CS field, and a non-zero statistical parameter $\theta$ which does not renormalize to zero at the one-loop level. Since $\theta$ does not renormalize at all beyond the one-loop level, this system will have an energy gap to all orders in perturbation theory. Consequently, perturbation theory about the saddle-point does not converge to anything which describes the original electron system, which is gapless.

This failure of perturbation theory about the saddle-point can be understood as follows. The Hilbert space for the original electron wavefunction is spanned by Slater determinants of $N$ electrons in the $2 N$ single-particle states in the lowest Landau level (ignoring Landau-level mixing). The electron wavefunction is thus a sum over $N$ products $\left(z_{i}-z_{j}\right)$ times exponential factors. Thus, the wavefunction vanishes as $\left(z_{i}-z_{j}\right)$ as two electrons are brought towards one another. We then perform the singular gauge transformation to obtain the equivalent boson wavefunction. The gauge transformation only changes the relative phases of the wavefunction, which will now contain factors $\left|z_{i}-z_{j}\right|$. The singular gauge transformation maps the original analytic electron wavefunction in the lowest Landau level onto a non-analytic boson wavefunction. Thus, the wavefunction so obtained does not have an expansion in states in the lowest Landau level alone. On the other hand, the starting point for perturbation theory is the homogeneous $\nu=1$ boson solution. The wavefunction of this state $\Phi_{1}$ for $\nu=1$ bosons in the large- $V_{0}$ limit is a product of factors $\left(z_{i}-z_{j}\right)$ and $z_{i}^{*}$, times the exponential factors, which has a completely different non-analytic structure. We therefore speculate that the failure of perturbation theory about the saddle-point corresponds to the fact that the $V_{0}$ boson wavefunction at $\nu=1$ is outside the radius of convergence of perturbation expansions about the (exact) non-analytic boson wavefunction.

In conclusion, we have argued that interacting hard-core bosons exhibit a $\nu=1 \mathrm{IQHE}$, contrary to expectations. As a consequence, perturbation theory about the saddle-point of the equivalent CS boson system at $\nu=1$ obtained by from a singular gauge transformation 
of an electron system at $\nu=1 / 2$ fails completely. The reason is that the saddle-point approximation describes an incompressible $\nu=1$ hard-core boson system with an energy gap. When fluctuations at the one-loop level about the saddle point are included, the statistical parameter remains non-zero, and it does not renormalize beyond the one-loop level. Therefore, the system has an energy gap and remains incompressible to all orders in perturbation theory. This shows explicitly that the rather crude saddle-point approximation can give rise to completely unphysical results.

We would like to thank the National Science Foundation for its support through grant DMR93-01433, and O.H. would also like to thank S. Östlund and M. Jonson at Chalmers University of Technology for their hospitality, where part of this work was done. 


\section{REFERENCES}

[1] For reviews of this topic, see, for example, Physics and Mathematics of Anyons, edited by S.S. Chern, C.W. Chu and C.S. Ting (World Scientific, Singapore, 1991), and Field Theories of Condensed Matter Systems, by E. Fradkin (Addison-Wesley, Redwood City 1991).

[2] B.I. Halperin, P.A. Lee, and N. Read, Phys. Rev. B 47, 7312 (1993).

[3] J.K. Jain and S. Rao, Mod. Phys. Lett. B, 9, 611 (1995).

[4] J. Jain, Phys. Rev. Lett. 63, 199 (1989); Phys. Rev. B 40, 8079 (1989); Phys. Rev. B 41, 7653 (1990).

[5] S.C. Zhang, T.H. Hansson and S. Kivelson, Phys. Rev. Lett. 62, 82 (1989).

[6] See, for example, S. Coleman and B. Hill, Phys. Lett. 159B, 184 (1985), and G.W. Semenoff, P. Sodano and Y.S. Wu, Phys. Rev. Lett. 62, 715 (1989).

[7] D.J. Thouless, M. Kohmoto, M.P. Nightingale and M. den Nijs, Phys. Rev. Lett. 46, 405 (1982); Q. Niu, D. Thouless and Y.S. Wu, Phys. Rev. B 31, 3372 (1985); M. Kohmoto, Ann. Phys. (New York) 160, 343 (1985).

[8] For a review, see A. Zee in High-temperature superconductivity, edited by K.S. Bedell, D. Coffey, D.E. Meltzer, D. Pines and J.R. Schrieffer (Addison-Wesley, Redwood City, CA, 1990), and Ref. [1].

[9] N. Read, Phys. Rev. Lett. 61, 86 (1989).

[10] D.-H. Lee in Physics and Mathematics of Anyons, edited by S.S. Chen, C.W. Chu, and C.S. Ting (World Scientific, Singapore, 1991).

[11] D. Schmeltzer, Phys. Rev. B 47, 11980 (1993).

[12] H.P Wei et al., Phys. Rev. Lett. 61, 1297 (1988). 
[13] L. Engel et al., Surf. Sci. 229, 13 (1990).

[14] S. Koch et al., Phys. Rev. Lett. 67, 883 (1991).

[15] V. Kalmeyr and S.C. Zhang, Phys. Rev. B 46, 9889 (1992).

[16] E. Rezayi and N. Read, Phys. Rev. Lett. 72, 900 (1994).

[17] R.L. Willett et al., Phys. Rev. Lett. 71, 3846 (1993); R.L. Willett et al.,, Phys. Rev. B 47, 7344 (1993).

[18] W. Kang et al., Phys. Rev. Lett. 71, 3850 (1993).

[19] V. J. Goldman, B. Su and J. K. Jain, Phys. Rev. Lett. 72, 2065 (1994).

[20] For an incomplete list, see, for example, A.M.M Pruisken, Phys. Rev. Lett. 61, 1297 (1988); D. Chklovskii and P.A. Lee, Phys. Rev. B 48, 18060 (1993); D.H. Lee, Z. Wang and S. Kivelson, Phys. Rev. Lett. 70, 4130 (1993); D.G. Polyakov and B.I. Shklovskii, Phys. Rev. Lett. 70, 3796 (1993); H.L. Zhao and S. Feng, Phys. Rev. Lett. 70, 4134 (1993); V. Kalmeyer, D. Wei, D.P. Arovas and S.C. Zhang, Phys. Rev. B 48, 11095 (1993).

[21] R.B. Laughlin, Phys. Rev. B 23, 5632 (1981); Phys. Rev. Lett. 50, 1395 (1983).

[22] B.I. Halperin, Phys. Rev. B 25, 2185 (1982).

[23] Field Theories of Condensed Matter Systems, E. Fradkin (Addison-Wesley, Redwood City, Ca, 1991). 